\title{
Cell Switch Off Technique Combined with Coordinated Multi-Point (CoMP) Transmission for Energy Efficiency in Beyond-LTE Cellular Networks
}

Gencer Cili, Halim Yanikomeroglu, and F. Richard Yu

Department of Systems and Computer Engineering, Carleton University, ON, Canada

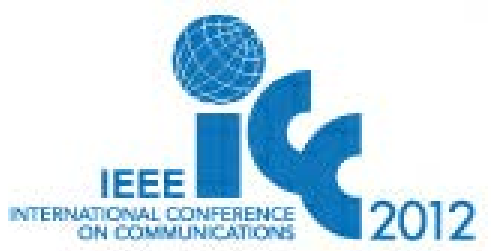




\section{Introduction}

- Energy Efficiency of Cellular Systems became a major performance metric:

- Increased use of cellular devices -> $\mathrm{CO}_{2}$ emission rise in cellular networks

- Information and Communications Technology is responsible for 2-10\% of global energy consumption

- Access stratum is responsible for $60-80 \%$ the whole cellular network energy consumption

- Energy Efficiency metric: Bits/Joule should be jointly considered with spectral efficiency metric

- Methods for Energy Efficient Access Networks:

- Energy efficiency in Base Stations

- Energy efficiency using Cooperative Base Station Schemes

- Energy Efficiency using renewable energy resources

- Energy efficiency via heterogonous networks

- Cognitive Radio \& Cooperative relaying for Energy Efficiency

- Our contributions

- LTE-A Downlink CoMP used jointly with traditional Cell Switch Off Schemes

- Model energy \& spectral efficiency of CoMP + Cell Switch Off Schemes

- Use DL CoMP to serve the users in switched off cell

- Demonstrate CoMP challenges: Estimation Errors + System Delays 


\section{Overview of Green Access Networks}

- Cell size adjustments according to traffic load fluctuations

- Cells with the low traffic zoom into zero, and the neighbor cells zoom out by physical adjustments

- Base stations with low Spectral Efficiency are turned off - Spectrally efficient BSs serve the users

- 24- hour traffic routine is analyzed, optimum cell switch off/on periods are found

- Ratio between the dynamic and the fixed power of a base station: Switch Off decision parameter

\section{Cell Switch Off Suggestion by Academia [6]:}

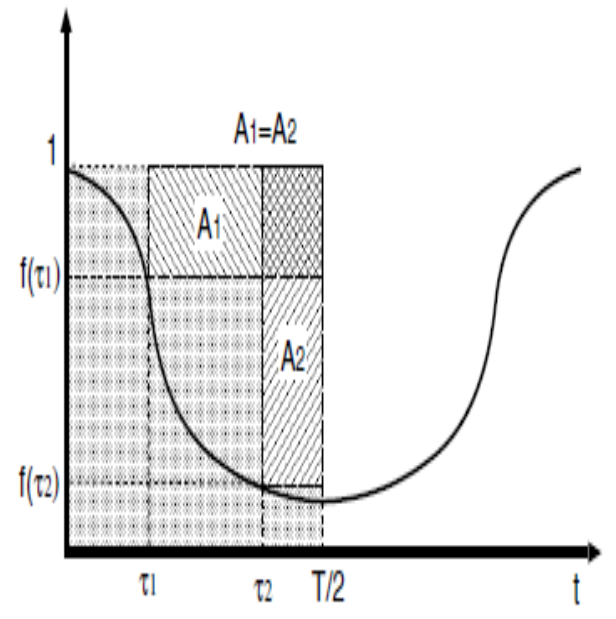

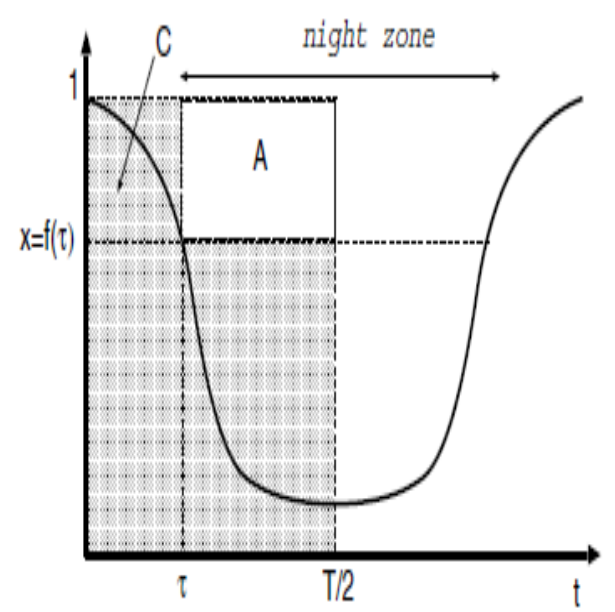

3GPP - Small Cell Switch Off Scheme [15]:

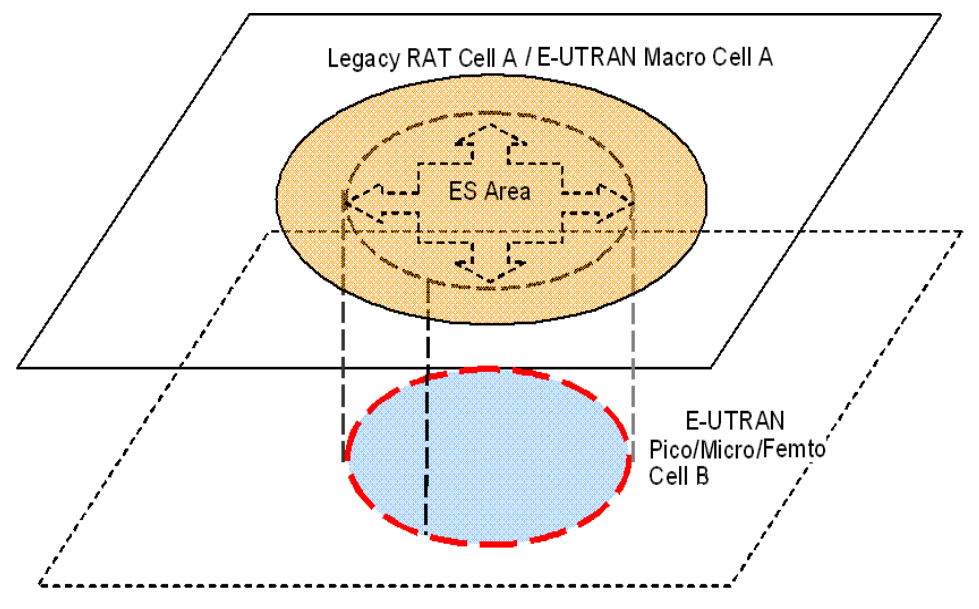

Proposal: Replace antenna tilt/Transmit power increase of active cells by DL CoMP to serve the users in the switched off cell. 


\section{LTE Downlink Transmission and CoMP Procedures}

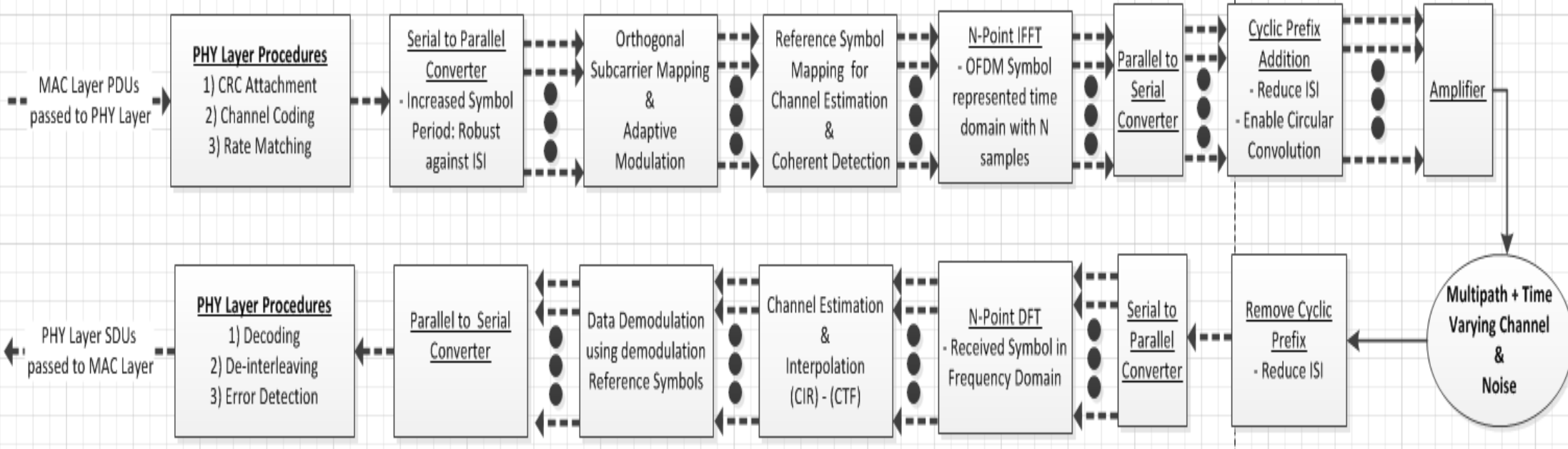

$$
\begin{aligned}
& y_{N x 1}=x_{N x N} h_{N x 1}+n_{N x 1} \\
& {\left[\begin{array}{c}
y_{1}(k) \\
\vdots \\
y_{N}(k)
\end{array}\right]=\left[\begin{array}{cccc}
x_{1}(k) & x_{N}(k) & \cdots & x_{2}(k) \\
\vdots & \vdots & \vdots & \vdots \\
x_{N}(k) & x_{N-1}(k) & \cdots & x_{1}(k)
\end{array}\right]\left[\begin{array}{c}
h_{1}(k) \\
\vdots \\
h_{N}(k)
\end{array}\right]+\left[\begin{array}{c}
n_{1}(k) \\
\vdots \\
n_{N}(k)
\end{array}\right]} \\
& y_{N x 1}=F_{N x N}{ }^{H} X_{N x N} F_{N x N} h_{N x 1}+n_{N x 1}
\end{aligned}
$$




\section{LTE Downlink Transmission and CoMP Procedures}

CoMP Definition: Dynamic coordination among multiple geographically separated points referred as CoMP cooperating set for downlink transmission and uplink reception

\section{Downlink CoMP Schemes:}

1) Joint Processing: User Plane Data available at each Transmission Point

2) Coordinated Scheduling/Coordinated Beamforming: User Plane Data @ Serving Cell

\section{CoMP Deployment Scenarios:}

1) eNB - eNB

2) $\mathrm{RRH}-\mathrm{RRH}$

3) eNB - High Power RRH

4) eNB - Low Power RRH

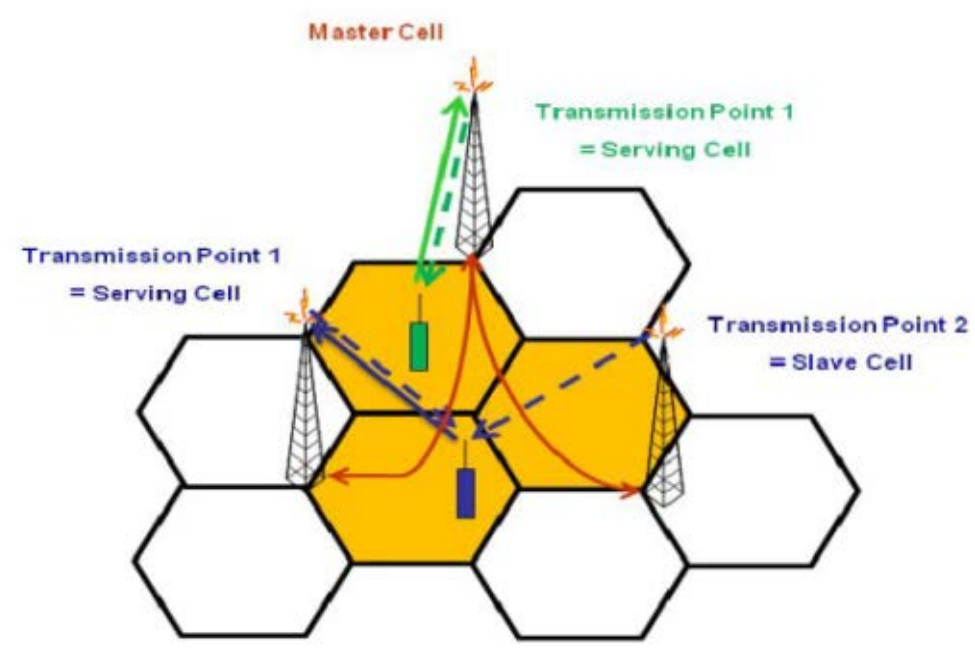




\section{CoMP + Cell Switch Off Model}

\section{Cellular Layout + Parameters:}

1) 19 eNBs with hexagonal layout

2) Center Cell Switched Off,

3) Remaining 18 eNBs are in CoMP Cooperating \& Measurement Set

4) Uniform user distribution in the switched off cell $i \in[1, \ldots, 500]$

5) Cooperating Cell IDs: $n \in[1, \ldots, 18]$

6) Channel samples every TTI according to Winner SCME model: $t \in[1, \ldots, 1000]$

7) Each UE-eNB link is modeled independently

8) Large scale path loss model according to ITU$\mathrm{R}$ report $\mathrm{M} .2135$

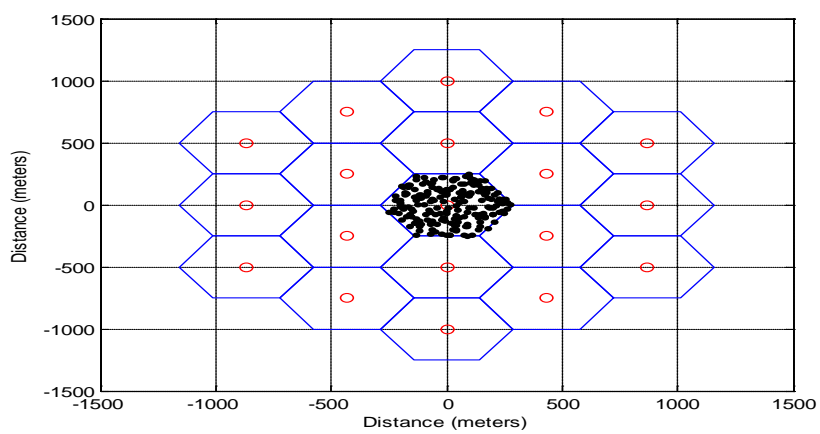

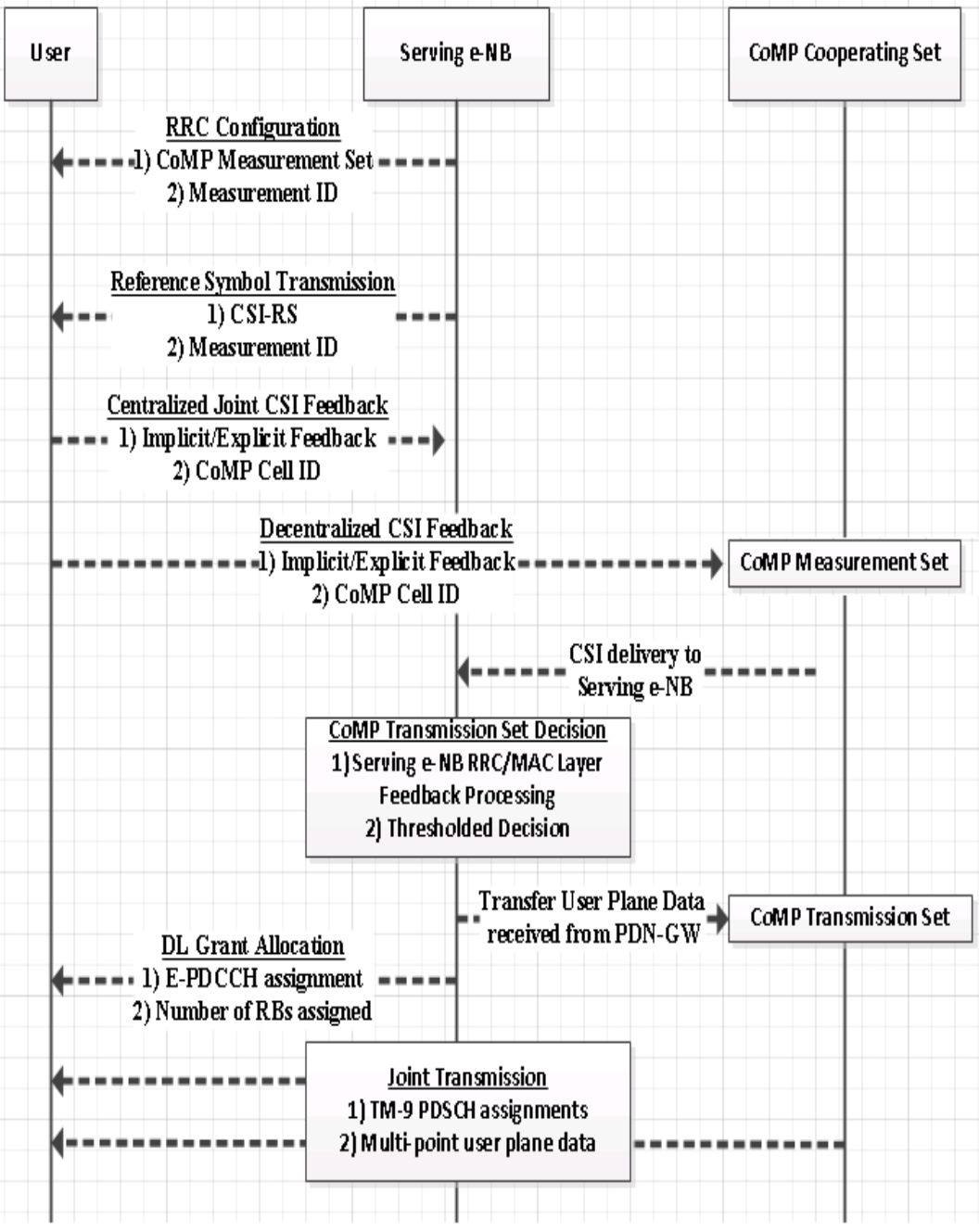




\section{CoMP + Cell Switch Off Model}

\section{CoMP Transmission Set Forming:}

- $\quad$ Serving cell configures the UE for multi-point measurements for each eNB in CoMP measurement set

- $\quad$ CSI-RS enables Multi-Point Channel Estimation

- Actual measured received power from eNB $n$ by user $i$ at TTI $t$ :

- $\quad P_{R X}(n, t, i)=P_{T X}(n)-P L(n, i)-P_{\text {Fading }}(n, i, t)$

- Implicit/Explicit multipoint channel feedback obtained at Serving Cell

- Received feedback due to estimation error + delay:

- $\quad P_{R X_{-} \text {err }}(n, t, i)=P_{R X}(n, t-\Delta, i)+P_{\text {err }}(\mu, \sigma)$

- Thresholded Decision to Form the CoMP Transmission Set: Serving Cell Power - Measured cell $\leq 3 \mathrm{~dB}$

- Time-varying CoMP Transmission Set: $J T(i, t)$

- Joint PDSCH transmission + Cross-point scheduling over certain time/frequency resources

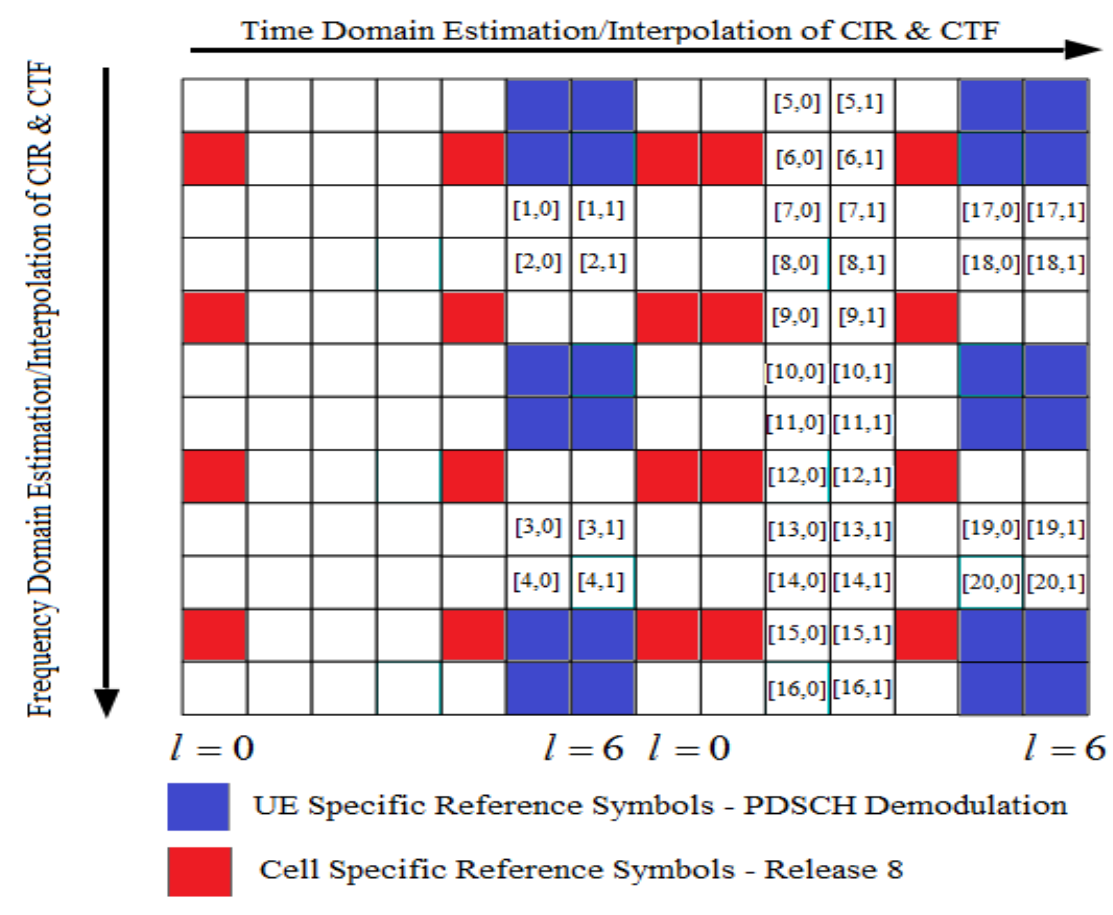

[Cell ID, Antenna Port]: CSI Reference Symbols - Release 10

Note: Release-8 devices use CRS for channel estimation (8 REs over RB pair), but Rel -11 CoMP channel estimation uses CSI-RS (1 RE over RB pair per antenna port) = Multi-point channel estimation is more vulnerable to channel estimation errors due to scarce REs to track the channel using autocorrelation functions 


\section{CoMP Performance Metrics - Capacity}

- Joint PDSCH transmission (TM-9) mitigates the Inter-cell Interference

Single Point Transmission

$\operatorname{SINR}=\frac{P_{\text {serving }}}{\sum_{i=1}^{K} P_{i}+P_{\text {Noise }}}$
CoMP Downlink Transmission

$$
\operatorname{SINR}_{\text {CoMP }}=\frac{P_{\text {serving }}+P_{j}+P_{m}}{\sum_{\substack{i=1 \\ i \neq j, m}}^{K} P_{i}+P_{\text {Noise }}}
$$

Total received Power from CoMP Transmission Set

$$
P_{J T}(i, t)=\sum_{n \in J T(i, t)} P_{R X}(n, t, i)
$$

Perceived Downlink Capacity due to CoMP

$$
C(i, t)=B W(i, t) * \log _{2}\left(1+\frac{P_{J T}(i, t)}{P_{\text {Noise }}+\sum_{n \notin J T(i, t)} P_{R X}(n, t, i)}\right)
$$

Note: CoMP transmission set $J T(i, t)$ is formed according to delayed and inaccurately estimated channel samples. $B W(i, t)$ is dependent on the number of RBs assigned to UE 


\section{CoMP Performance Metrics - Energy Efficiency}

\section{CoMP Power Consumption Model}

Signal Processing Power

Backhauling Power

Additional Data capacity for CoMP Backhauling

Total Power Consumption of an eNB using CoMP

$$
P_{S P-C o M P}=58 *\left(0.87+0.1 N_{C}+0.03 N_{C}^{2}\right)
$$

$$
\begin{aligned}
P_{B H} & =\frac{C_{B H}}{100 \mathrm{Mbits} / \mathrm{sec}} * 50 \mathrm{~W} \\
C_{B H} & =\frac{N_{c^{*}}\left(2 N_{C}\right) * p * q}{T_{S}} \mathrm{bits} / \mathrm{sec}
\end{aligned}
$$

$$
P_{\text {CoMP }}=N_{S} * N_{\frac{P A}{\text { sector }}} *\left(\frac{P_{T X}}{P A_{\text {eff }}}+P_{S P}\right)\left(1+C_{C}\right)\left(1+C_{B B}\right)+P_{B H}
$$

\section{Power Consumption Parameters}

$N_{S}=$ Number of Sectors $N_{\frac{P A}{\text { sector }}}=$ Power amplifiers per sector $P_{T X}=$ DL Transmit Power, $C_{C}=$ Cooling Loss $C_{B B}=$ Battery Backup $N_{C}=$ Number of points in Joint Transmission $p=$ pilot density $q=$ CSI signalling $T_{S}=$ Symbol Period $P A_{e f f}=$ Power Amplifier Efficiency 


\section{CoMP Performance Metrics - Energy Efficiency}

$$
\text { Energy Efficiency }=\frac{\text { Capacity }(\text { bits } / \text { sec })}{\text { Power Consumption }(\text { Joules } / \text { sec })}=\text { bits } / \text { Joule }
$$

\section{Time Varying Energy Efficiency}

Joint Transmission CoMP Operation $\left(N_{C} \geq 2\right) \quad E E(i, t)$

$$
=\frac{C(i, t)}{P_{\text {CoMP }}+\left(N_{J T(i, t)}-1\right) *\left(P_{\text {CoMP }}-P_{\text {Base }}\right)}
$$

Single Point Transmission $\left(N_{C}=1\right)$

$$
E E(i, t)=\frac{C(i, t)}{P_{\text {Base }}}
$$

\section{Notes:}

1) $P_{B a s e}$ has $P_{B H}=0$ since there is not need for multi-point CSI transfer to serving cell

2) $P_{S P-C o M P}=58 \mathrm{~W}$ since $N_{C}=1$ 


\section{Propagation Model - Large Scale Pathloss}

$$
\begin{aligned}
& P L_{L o S}(d B)=22 \log _{10} d+28+20 \log _{10} f_{c}, 10 m<d<d_{B P} ; \\
& P L_{L o S}(d B)=40 \log _{10} d+7.8+2 \log _{10} f_{c}-18 \log _{10} h_{B S}-\log _{10} h_{U T}, d_{B P}<d<5000 m ; \\
& P L_{N L O S}(d B)= \\
& 161.04-7.1 \log _{10} L+7.5 \log _{10} h_{B}-\left(24.37-3.7\left(\frac{h}{h_{B S}}\right)^{2}\right) * \log _{10} h_{B S}+\left(43.42-3.1 \log _{10} h_{B S}\right) * \\
& \left.\left(\log _{10} d-3\right)+20 \log _{10} f_{c}-\left(3.2\left(\log _{10} 11.75 h_{U T}\right)\right)^{2}-4.97\right) ; \\
& \operatorname{Prob}(L o S)=\min \left(\frac{18}{d}, 1\right) *\left(1-e^{-\frac{d}{63}}\right)+e^{-\frac{d}{63}} .
\end{aligned}
$$

\begin{tabular}{|c|c|}
\hline Parameter & Value \\
\hline Carrier Frequency $\left(f_{c}\right)$ & $2110 \mathrm{Mhz}$ \\
\hline BS (Base Station) Antenna Height & $24 \mathrm{~m}$ \\
$\left(h_{B S}\right)$ & \\
\hline User Terminal Antenna Height $\left(h_{U T}\right)$ & $0.5 \mathrm{~m}$ \\
\hline Average Street Width $(\mathrm{L})$ & $20 \mathrm{~m}$ \\
\hline Average Building Height $\left(h_{B}\right)$ & $20 \mathrm{~m}$ \\
\hline LoS Shadowing $\left(\sigma_{L O S}\right)$ & $4 \mathrm{~dB}$ \\
\hline NLoS Shadowing $\left(\sigma_{N L O S}\right)$ & $6 \mathrm{~dB}$ \\
\hline Break Point Distance $\left(d_{B P}\right)$ & $337.6 \mathrm{~m}$ \\
\hline Transmission Power $\left(P_{T X}\right)$ & $20 \mathrm{~W}$ \\
\hline
\end{tabular}

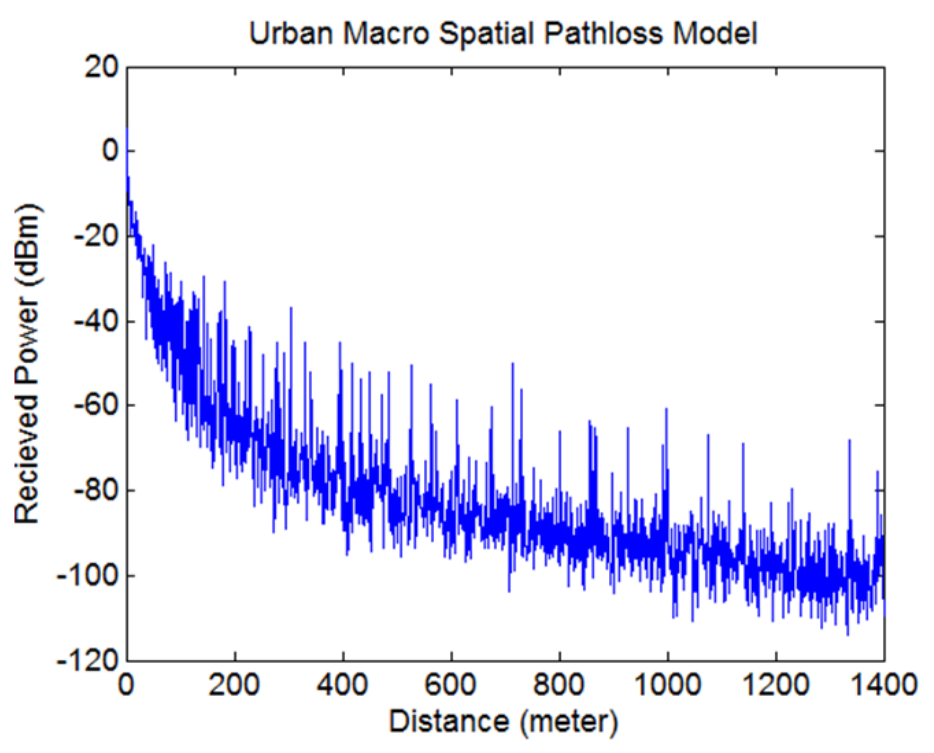




\section{Propagation Model - Small Scale Fading}

Complex Baseband Channel Impulse Response:

$h_{n, i}\left(t, \tau_{l}\right)=A_{l}(t) e^{j 2 \pi f_{d_{l}}(t)} e^{j 2 \pi f_{c} \tau_{l}} e^{j 2 \pi \emptyset_{l}} \delta(\tau$ $\left.-\tau_{l}\right)$

\section{Received Multipath Signal in Time Domain}

$s(t)=\sum_{l=0}^{L} A_{l} * \cos \left(2 \pi f_{c} t+2 \pi f_{d_{l}} t+\phi_{l}\right)$

Received Power Change Due to Small Scale Fading

$P_{\text {Fading }}(n, i, t)=10 * \log _{10}\left[\left(\frac{\left|\sum_{l=1}^{L} h_{n, i}(t, \tau)\right|}{2}\right)^{2}\right]$

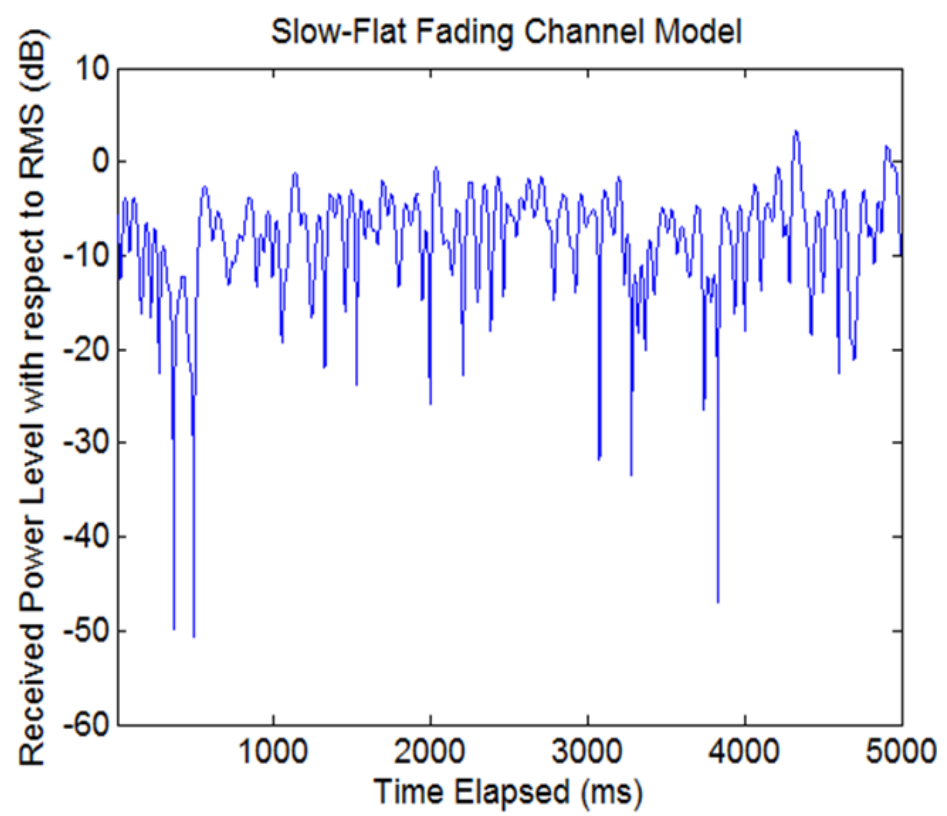

- Complex multipath components go through summation due to the narrow band nature of OFDMA

- Each UE-CoMP measurement set member have independent channels

- Main contributors to the multipath phase are $f_{d_{l}}$ and $\emptyset_{l} ; 2 \pi f_{c} \tau_{l}$ is due to difference of propagation of each multipath delay tap 


\section{Traditional Cell Switch Off vs. CoMP Aided Scheme}
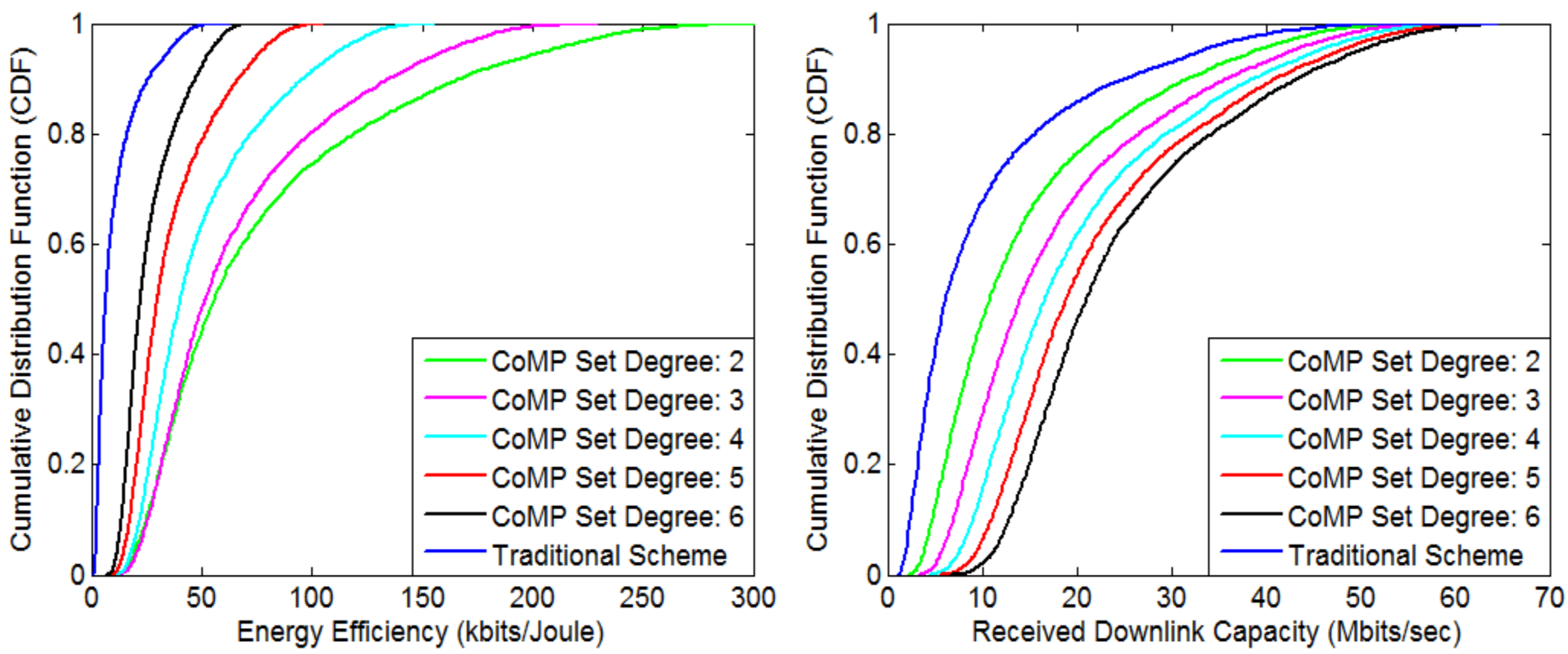

- CoMP aided schemes yield both spectrally and energy efficient systems compared to traditional cell switch off schemes

- Increased CoMP Transmission Set Degree improves the DL capacity due to mitigated ICI

- Non-adaptive increase in CoMP set degree decreases the energy efficiency of the system since the gained capacity is not worth the power consumption overhead

- Proof of Concept: Serving cell needs an adaptive thresholded decision mechanism for Joint PDSCH transmission set clustering. 


\section{Performance Analysis of CoMP Schemes subject to Inaccurate Clustering}

\section{Performance Subject to Multi-Point Channel Estimation Errors}
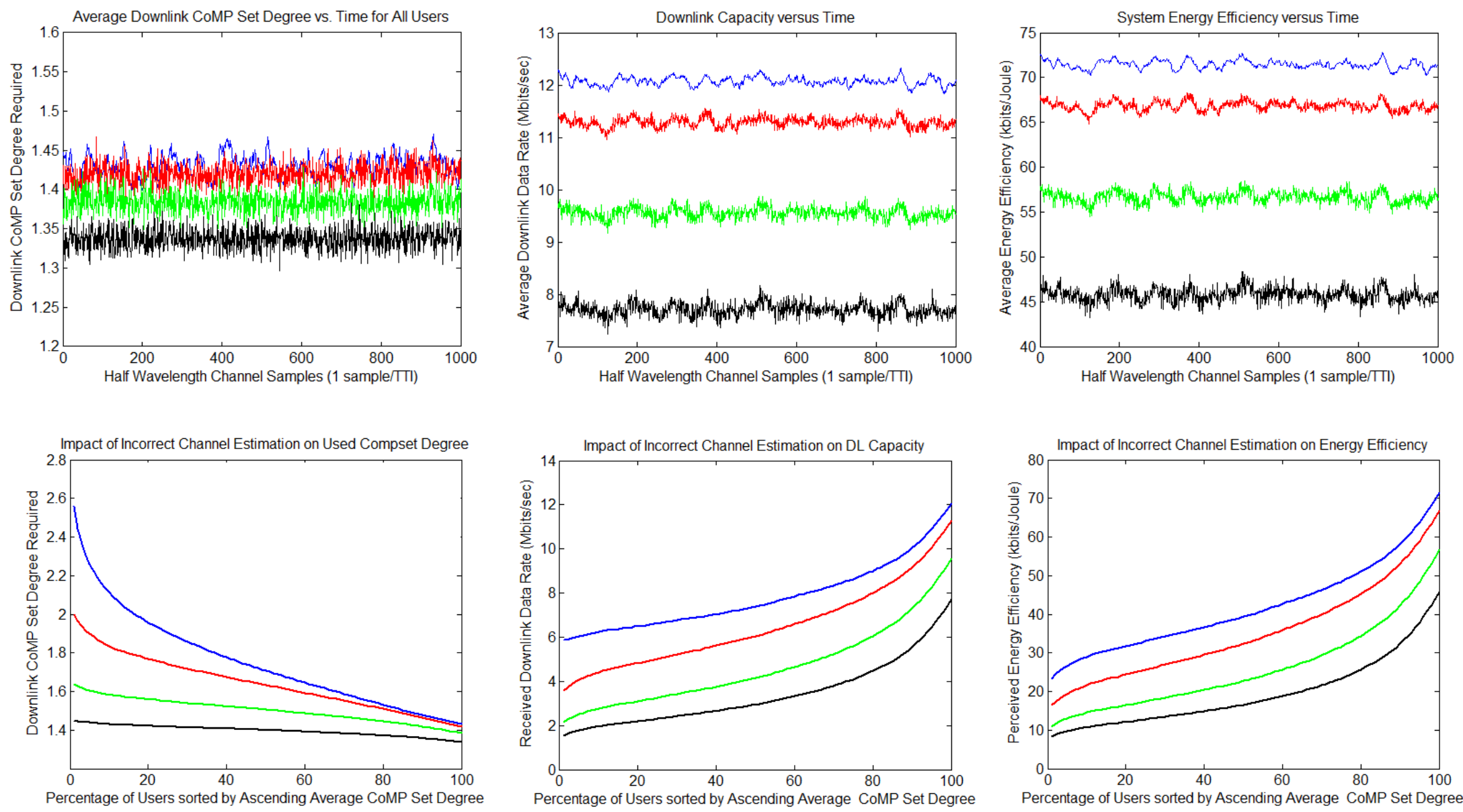


\section{Performance Degradation Due to Multi-Point Channel Estimation Errors}

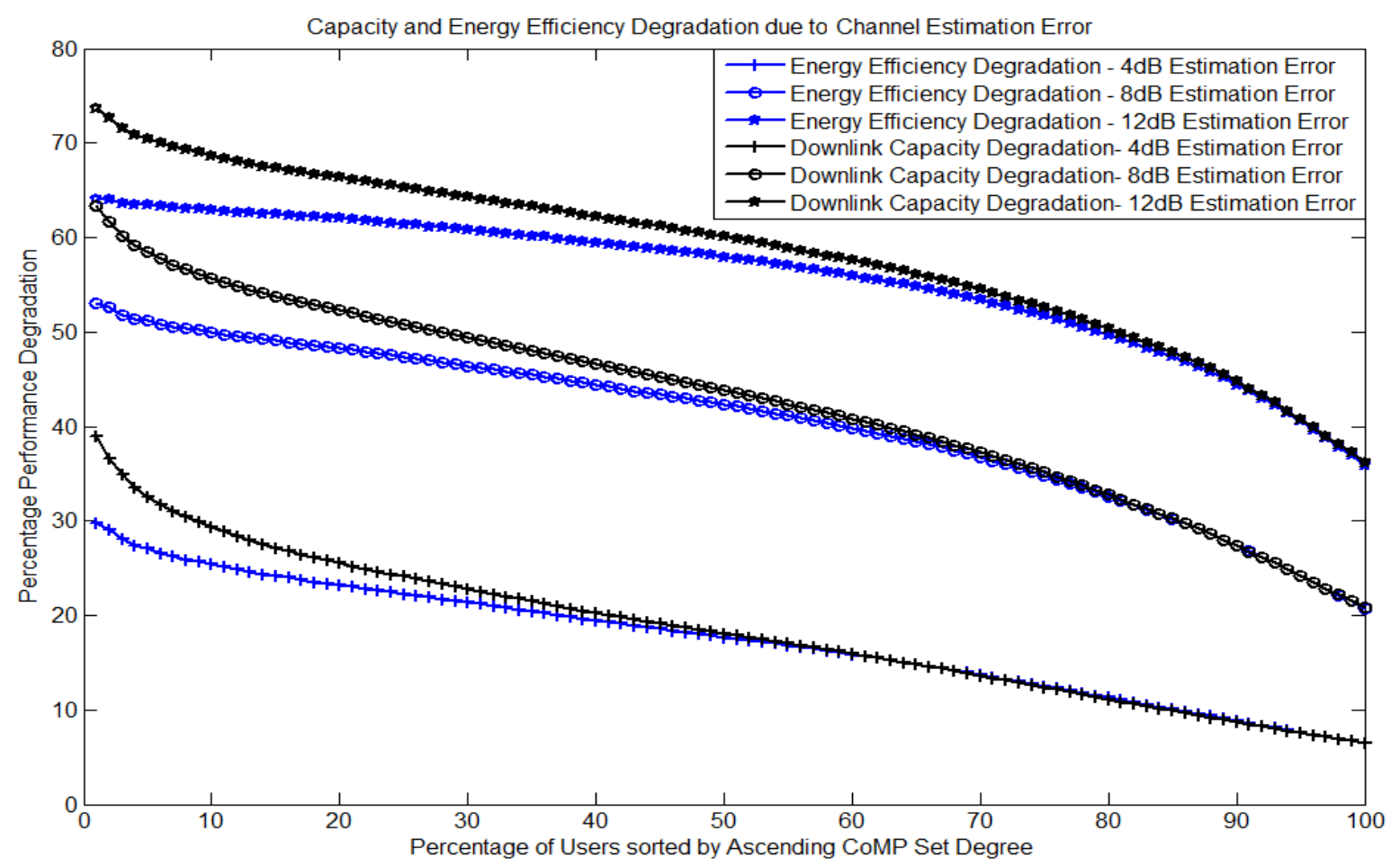

- $\quad P_{R X_{-} e r r}(n, t, i)=P_{R X}(n, t-\Delta, i)+P_{\text {err }}(\mu, \sigma)$

- Users that require higher Joint Transmission CoMP sets get affected the most

- Channel Estimation Errors decrease the used CoMP set degree, eNBs that are supposed to be part of joint transmission get down-selected due to estimation errors

- Energy Efficiency and Capacity get affected differently since Capacity degradation is dependent on CoMP set degree and the choice of points however energy efficiency is reliant on capacity/power trade-off where power consumption is purely dependent on the set degree 


\section{Performance Degradation Due to CoMP System Delay}

\section{CoMP Clustering Decision Delay are due to:}

1) Exchange of multi-point CSI feedback between the CoMP measurement set members and the serving cell (anchor) - lack of aggregate feedback

2) Network Topology Limitations causing latency

3) Node Processing \& Decision Delay:

- Received Power Estimation

- Sorting the members of CoMP measurement set

- Thresholded Decision

4) Exchange of User Plane payload between the transmission points

- $R_{h}\left(\Delta \boldsymbol{\tau}_{\boldsymbol{M}}, \tau_{l}\right)=\left[\begin{array}{ccc}E\left[h\left(t_{1}, \tau_{l}\right) h\left(t_{1}, \tau_{l}\right)^{*}\right] & \cdots & E\left[h\left(t_{1}, \tau_{l}\right) h\left(t_{N}, \tau_{l}\right)^{*}\right] \\ \vdots & \ddots & \vdots \\ E\left[h\left(t_{N}, \tau_{l}\right) h\left(t_{1}, \tau_{l}\right)^{*}\right] & \cdots & E\left[h\left(t_{N}, \tau_{l}\right) h\left(t_{N}, \tau_{l}\right)^{*}\right]\end{array}\right]$

- $\operatorname{Prob}\left(\left|h\left(t_{i}, \tau_{0}\right)-h\left(t_{j}, \tau_{0}\right)\right|>\varepsilon\right) \leq$ $2\left(R_{h}(|\Delta t=0, \Delta \tau=0|)-R_{h}\left(\left|t_{i}-t_{j}, \Delta \tau=0\right|\right)\right) / \varepsilon^{2}$

- $R_{h}\left(\Delta \boldsymbol{\tau}_{M}, \tau_{l}\right)$ is a decreasing function with a maximum value at $R_{h}\left(0, \tau_{l}\right)$

- Increased System delay or High Doppler shift will yield inaccurate clustering 


\section{Performance Degradation Due to CoMP System Delay}

Performance degradation under low mobility conditions $(v=6 \mathrm{~km} / \mathrm{h})$

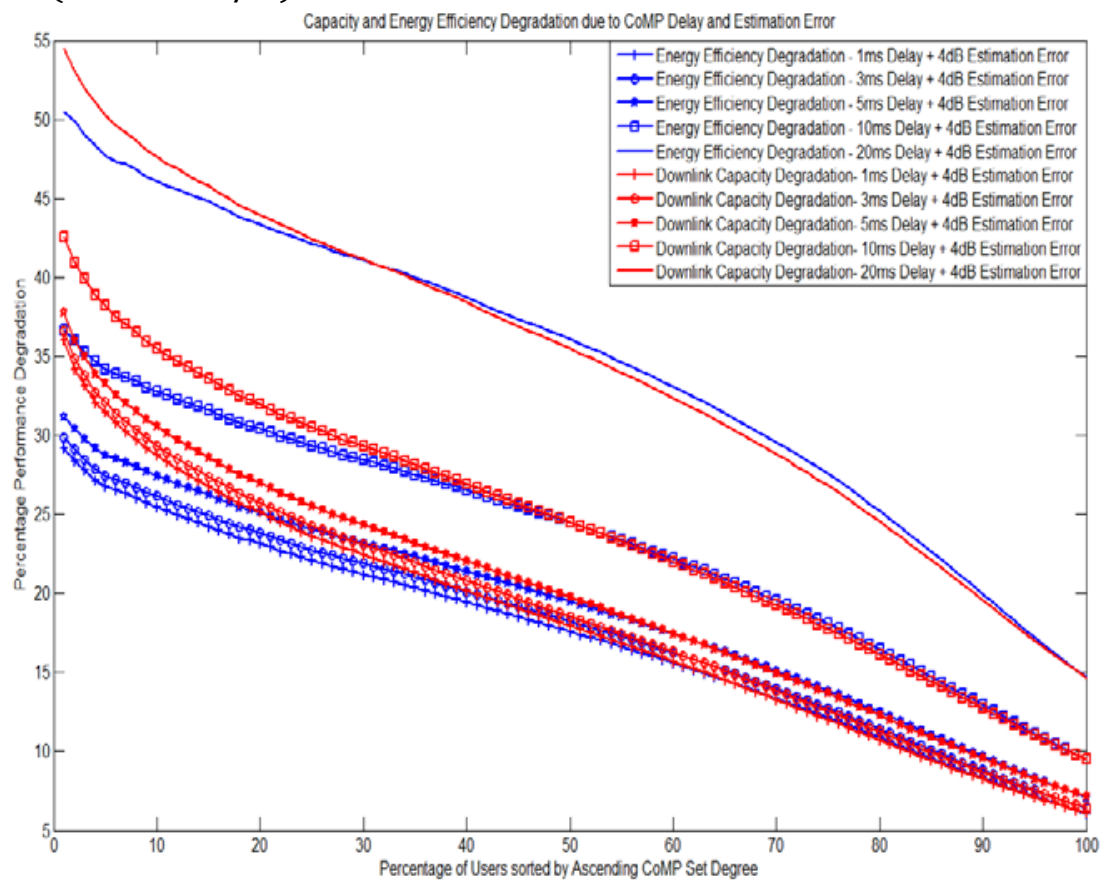

Performance degradation under high mobility conditions $(v=120 \mathrm{~km} / \mathrm{h})$

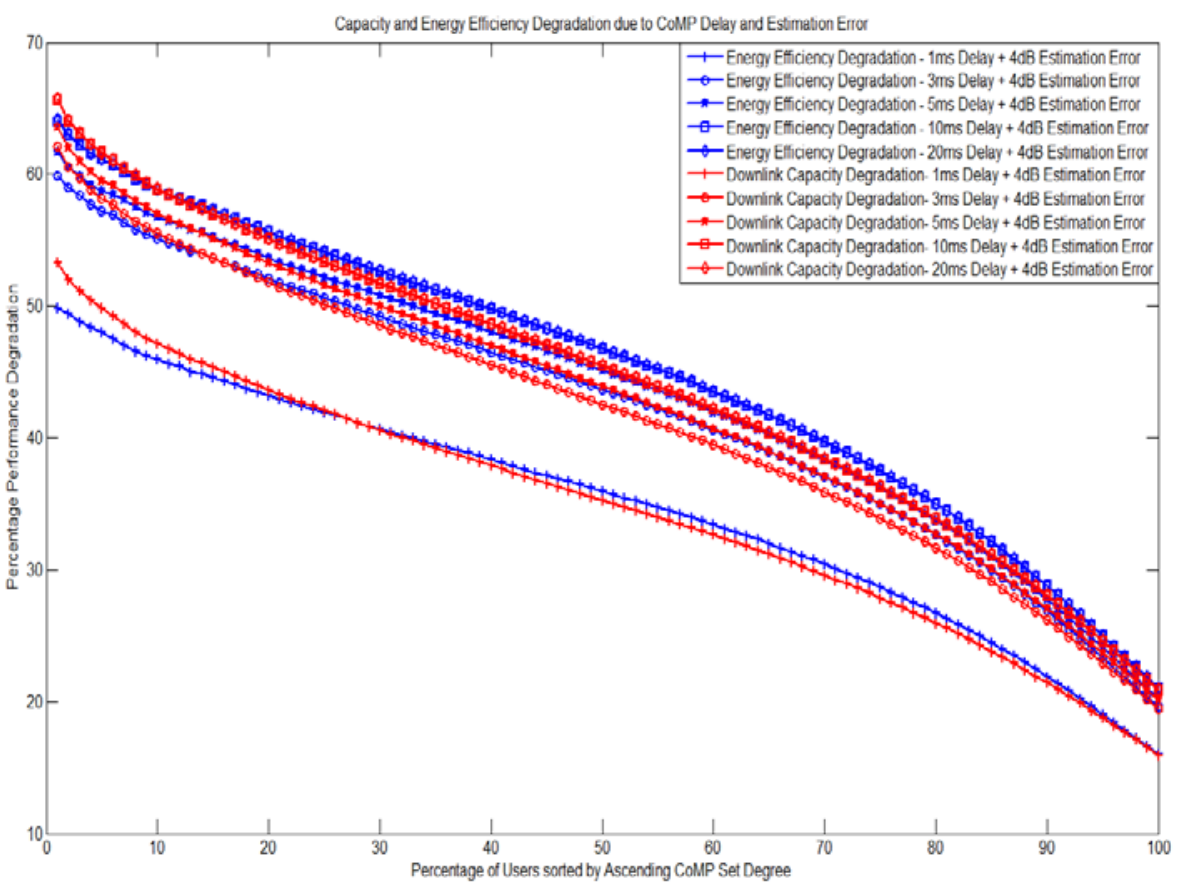

- For low coherence time scenarios, CoMP clustering gets affected severely

- Multi-point channel estimation errors had a direct impact on decreasing the used joint transmission set degree, whereas system delays create performance degradation just by changing the members of the CoMP set for the same set degree 


\section{CONCLUSION \& SUMMARY}

- CoMP aided cell switch off schemes yield both energy and spectra efficient systems

- Unneccessary increase CoMP set degree decreases the energy efficiency of the system

- Capacity/Power trade-off for the CoMP systems are achieved by Serving cell thresholding decision for joint PDSCH transmission set clustering

- Traditional Cell switch off schemes have the challenge of proper traffic routine modeling

- CoMP aided Cell Switch Off schemes are dependent on CSI feedback \& clustering decision accuracy

- Multi-point channel estimation errors degrade the performance by decreasing the overall average CoMP set degree

- CoMP system delays degrade the performance by inaccurate choice of transmission set points while keeping the set degree same

- Inter-eNB deployment scenario suffers from faulty clustering under High Doppler conditions 


\section{Future Work}

- Time-varying nature of each multipath delay for each point mentioned in CoMP measurement set should be tracked individually using estimation/interpolation filter for CIR

- Tracking will be dependent on multi-point channel estimation each TTI and interpolation of the results over various subframes

- Estimation Filter length M should be adapted according to the CoMP set degree observed, especially for low mobility cases that has high CoMP set degrees increased filter length will improve the performance significantly

- $\tilde{h}_{t_{n}, \tau_{l}}=$

$$
\left[\left(R_{h}\left(\Delta t, \tau_{l}\right)+\sigma_{\text {noise }}^{2} I_{M x M}\right)^{-1} r_{h}\left(\Delta t, \tau_{l}\right)\right]^{H} \widehat{\boldsymbol{h}}_{t_{n, ., n-M+1} ; \tau_{l}}
$$

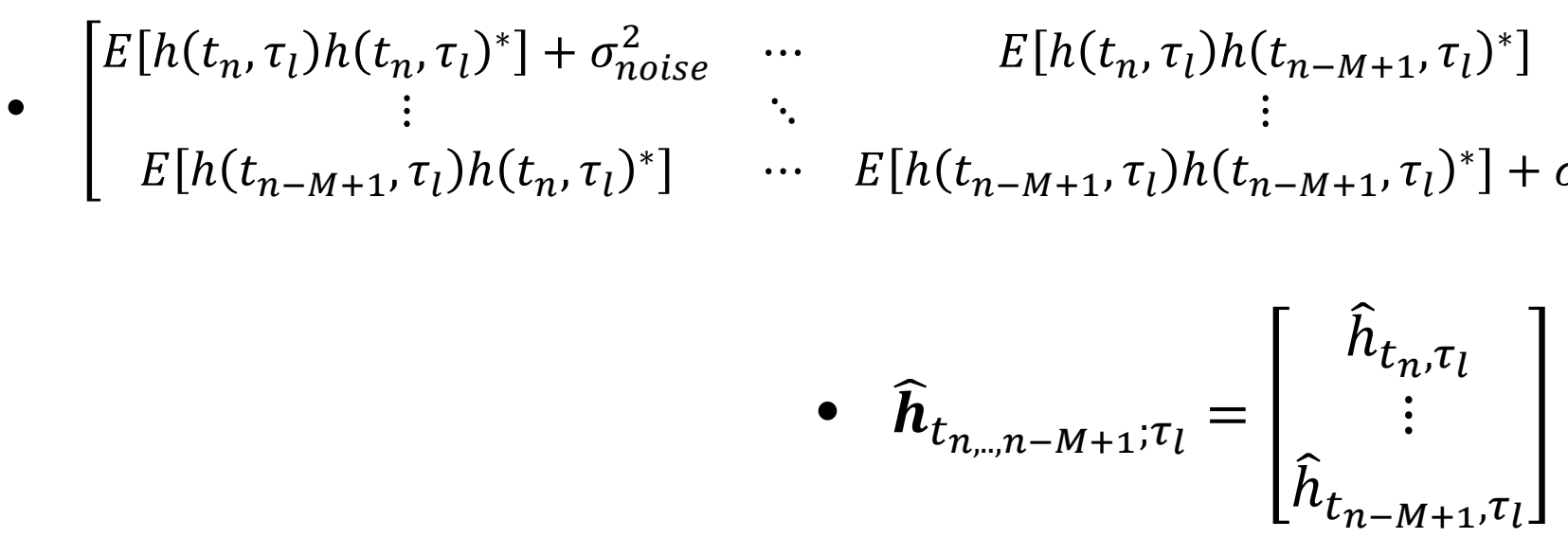




\section{REFERENCES}

[1] Global Action Plan, An inefficient truth, http $/ / /$ www globalactionplan_org_uk/, Report, 2007

[2] Z. Hashmi, H. Boostanimehr, and V. Bhargava, "Green cellular network: A survey, some research issues and challenges," IEEE Communications Surveys \& Tutorials, vol. 13, no. 4, pp. 524-540, Fourth Quarter 2011

[3] R. Schoenen, G. Bulu, A. Mirtaheri, and H. Yanikomeroglu, "Green communications by demand shaping and User-in-the-Loop tariff-based control" in Proc 2011 IEEE Online Green Communications Conference (IEEE GreenCom'1 I), Online, 2011

[4] Z. Niu, Y. Wu, J. Gong, and Z. Yang, "Cell zooming for cost-efficient green cellular networks," IEEE Commun. Mag., vol. 48, no. 11 , pp. 74-79, Nov. 2010 .

[5] M. Marsan, L. Chiaraviglio, D. Ciullo, and M. Meo, "Optimal energy savings in cellular access networks," in Proc. ICC'O9 Workshop on Green Commun., Dresden, Germany, June 2009.

[6] M. Marsan and M. Meo, "Energy efficient wireless Internet access with cooperative cellular networks." Computer Networks, vol. 55(2), pp. 386-398, Feb. 2011

[7] L. Chiaraviglio, D. Ciullo, M. Meo, and M. Marsan, "Energy-aware UMTS access networks," in Proc. IIth Int'l Symposium on Wireless Personal Multimedia Communications, Sept. 2008.

[8] L. Xiang, F. Pantisano, R. Verdone, X. Ge, and M. Chen, "Adaptive traffic load-balancing for green cellular networks," in PrOC. IEEE PIMRC'I1, Toronto, ON, Canada, Sept. 2011

[9] D. Cao, S. Zhou, C. Zhang, and Z. Niu, "Energy saving performance comparison of coordinated multi-point transmission and wireless relaying " in Proc. IEEE Globecom'10, Miami, FL, Dec. 2010.

[10] 3GPP TR 36.814 V1.2.1, "Further Advancements for EUTRA: Physical Layer Aspects," June 2009 .

[11] S. Brueck, L. Zhao, J. Giese, and M. Amin, "Centralized scheduling for joint transmission coordinated multi-point in LTE-Advanced," in Proc. 2010 International ITG Workshop on Smart Antennas, pp. 177-184, Feb. 2010.

[12] A. J. Fehske, P. Marsch, and G. P. Fettweis, "Bit per joule efficiency of cooperating base stations in cellular networks," in ProC. IEEE Globecom "IO Workshops, Miami, FL, Dec. 2010.

[13] O. Arnold, F. Richter, G. Fettweis, and O. Blume, "Power consumption modeling for different base station types in heterogeneous cellular networks," in Proc. Future Network and Mobile Summit $2010, \mathrm{pp}$. 1-8, June 2010.

[14] International Telecommunication Union, "Guidelines for evaluation of radio interface technologies for IMT-Advanced" Rep. ITU-R M.2135, 2008 .

[15] J. Salo, G. Del Galdo, J. Salmi, P. Kyösti, M. Milojevic, D. Laselva, and C Schneider. MATLAB Implementation of the 3GPP Spatial Channel Model (3GPP TR 25.996) [Online]. http://www.tkk.fi/Units/Radio/scm/ (Jan 2005)

[16] M. Sawahashi, Y. Kishiyama, A. Morimoto, D. Nishikawa, and M. Tanno, "Coordinated multipoint transmission/reception techniques for LTEadvanced," IEEE Wireless Commun, vol. 17, no. 3, pp. 26-34, Jun. 2010. 


\section{THANK YOU!}

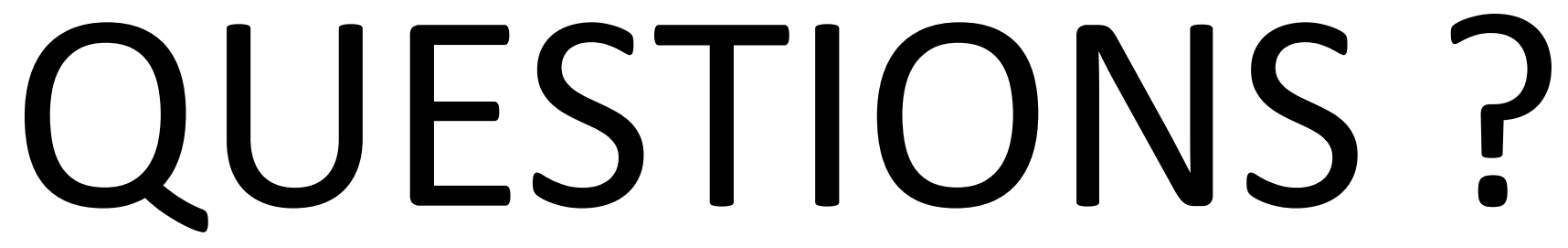

